have potential roles in disease pathologies. This will lay the groundwork for more successful therapeutic interventions by targeting multiple candidate molecules, such as a-synuclein and tau in diseases.

\section{ACKNOWLEDGEMENTS}

We thank all the members of Kayed laboratory for their helpful insights, suggestions and support. This work was supported by the Michael J. Fox foundation, the Gillson Longenbaugh Foundation, the Mitchell Center for Neurodegenerative Diseases, the Sealy Center for Vaccine Development and the NIH grants AG054025 (R.K.), NS094557 (R.K.) and AG055771 (R.K.).Competing interests: The authors declare no competing interests.

Publisher's note: Springer Nature remains neutral with regard to jurisdictional claims in published maps and institutional affiliations.

\section{REFERENCES}

1. Ahmed RM, Devenney EM, Irish M, Ittner A, Naismith S, Ittner LM, et al. Neuronal network disintegration: common pathways linking neurodegenerative diseases. J Neurol Neurosurg Psychiatry. 2016;87:1234-41.

2. Gerson JE, Mudher A, Kayed R. Potential mechanisms and implications for the formation of tau oligomeric strains. Crit Rev Biochem Mol Biol. 2016;51:482-96.

3. Sengupta U, Guerrero-Munoz MJ, Castillo-Carranza DL, Lasagna-Reeves CA, Gerson JE, Paulucci-Holthauzen AA, et al. Pathological interface between oligomeric alphasynuclein and tau in synucleinopathies. Biol Psychiatry. 2015;78:672-83.

4. Castillo-Carranza DL, Guerrero-Munoz MJ, Sengupta U, Gerson JE, Kayed R. alphaSynuclein Oligomers Induce a Unique Toxic Tau Strain. Biol Psychiatry 2018;84:499-508.

5. Spires-Jones TL, Attems J, Thal DR. Interactions of pathological proteins in neurodegenerative diseases. Acta Neuropathol. 2017;134:187-205.

6. Guerrero-Munoz MJ, Castillo-Carranza DL, Krishnamurthy S, Paulucci-Holthauzen AA, Sengupta U, Lasagna-Reeves CA, et al. Amyloid-beta oligomers as a template for secondary amyloidosis in Alzheimer's disease. Neurobiol Dis. 2014;71:14-23.

\title{
Ovarian hormones, genes, and the brain: the case of estradiol and the brain-derived neurotrophic factor (BDNF) gene
}

\author{
Shau-Ming Wei ${ }^{1,2}$ and Karen F. Berman ${ }^{1}$ \\ Neuropsychopharmacology (2019) 44:223-224; https://doi.org/10.1038/s41386-018-0223-5
}

The need for novel therapeutics has fueled the burgeoning impetus to uncover biological mechanisms that contribute to sex and individual differences in the manifestations of neuropsychiatric disorders. Investigating interactions between sex hormones and genetic makeup holds promise for this quest.

Animal studies have clearly established that sex hormones impact brain organization and function at critical developmental periods, including gestation and puberty; the neuromodulatory effects of ovarian steroids are well documented across the lifespan. However, little is known about the genesis of individual differences in cognitive and behavioral response to these hormones in humans. For example, why do some women develop postpartum depression while others do not, even in the face of the same hormonal events? Because ovarian hormones are important transcriptional regulators, their actions on the brain may vary according to individual differences in genetic make-up, suggesting an important research direction that could provide information regarding this and similar clinical questions.

The potential importance of such investigations is supported by preclinical studies in female transgenic mice harboring the uniquely human $B D N F$ Val ${ }^{66}$ Met variant. The $B D N F$ gene is of particular interest for investigating gene-hormone interaction because estradiol induces BDNF expression that mediates hippocampal function [1]. Likely as a consequence, in female $B D N F_{\text {Met }}$ knock-in mice, the estrus cycle critically interacts with the Val ${ }^{66}$ Met variant to modulate anxiety-related behaviors [2] as well as hippocampally dependent function and behavior [3].

Building on these preclinical experiments, we used two different but complementary neuroimaging modalities, the blood-oxygen- level depndent functional magnetic resonance imaging and positron emission tomography regional cerebral-blood flow techniques, to measure working memory-dependent brain function in healthy, regularly menstruating women during a 6-month hormone-manipulation protocol with three hormone conditions: ovarian suppression induced by the gonadotropin-releasing hormone agonist leuprolide acetate (Lupron), Lupron + estradiol replacement, and Lupron + progesterone replacement. We found a genotype-hormone interaction in the hippocampus, a region that is typically not recruited and is often even deactivated during working memory: in women carrying the $B D N F_{\text {Met }}$ variant, the hippocampus was atypically activated (i.e., abnormally recruited), but only in the presence of estradiol [4]. The results were consistent between both imaging platforms, providing important confirmatory data. Our findings demonstrate an estrogen sensitivity in the context of the Met variant in women, and thus provide an important translational step by demonstrating that the BDNF genotype-ovarian steroid interaction impacts neural function.

These studies offer evidence that harboring a genetic predisposition regulated in part by sex hormones in the brain, such as the $B D N F_{\text {Met }}$ allele, may have clinical implications [5]. Additionally, a recent preclinical study showing $B D N F_{\text {Met }}$ variant-specific elimination of hippocampal function during peri-adolescence [6] suggests the importance of future studies examining gene-hormone interactions during this critical period of brain development and hormonal change.

Delineating how the interplay between genes and sex hormones influences the brain has important relevance for women's mental health, for understanding individual differences

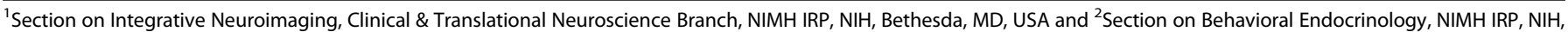
Bethesda, MD, USA

Correspondence: Karen F. Berman (bermank@mail.nih.gov)
}

Published online: 4 October 2018 
in hormonal effects on brain and behavior, and for revealing mechanisms that confer sex-related differential risk for neuropsychiatric disorders. Moreover, the potential importance of such gene-hormone interactions suggests a general strategy for further exploration that may inform individualized treatments.

\section{FUNDING AND DISCLOSURE}

This work was supported by the Intramural Research Program of the National Institute of Mental Health, National Institutes of Health. The authors declare no competing interests.

\section{REFERENCES}

1. McEwen BS, Akama KT, Spencer-Segal JL, Milner TA, Waters EM. Estrogen effects on the brain: actions beyond the hypothalamus via novel mechanisms. Behav Neurosci. 2012;126:4-16.
2. Bath KG, Chuang J, Spencer-Segal JL, Amso D, Altemus M, McEwen BS, et al. Variant brain-derived neurotrophic factor (Valine66Methionine) polymorphism contributes to developmental and estrous stage-specific expression of anxiety-like behavior in female mice. Biol Psychiatry. 2012;72:499-504.

3. Spencer JL, Waters EM, Milner TA, Lee FS, McEwen BS. BDNF variant Val66Met interacts with estrous cycle in the control of hippocampal function. Proc Natl Acad Sci USA. 2010;107:4395-4400.

4. Wei SM, Baller EB, Kohn PD, Kippenhan JS, Kolachana B, Soldin SJ, et al. Brainderived neurotrophic factor Val66Met genotype and ovarian steroids interactively modulate working memory-related hippocampal function in women: a multimodal neuroimaging study. Mol Psychiatry. 2017;23:1066.

5. Epperson CN, Bale TL. BDNF Val66Met polymorphism and brain-derived neurotrophic factor levels across the female life span: implications for the sex bias in affective disorders. Biol Psychiatry. 2012;72:434-6.

6. Giza Jl, Kim J, Meyer HC, Anastasia A, Dincheva I, Zheng $\mathrm{Cl}$, et al. The BDNF Val66Met prodomain disassembles dendritic spines altering fear extinction circuitry and behavior. Neuron. 2018;99:163-78.

\title{
Sex differences in the incidence of antidepressant-induced mania (AIM) in bipolar disorders
}

\author{
Aislinn Williams ${ }^{1}$ and Melvin G. Mclnnis (iD) ${ }^{2}$ \\ Neuropsychopharmacology (2019) 44:224-225; https://doi.org/10.1038/s41386-018-0216-4
}

Bipolar disorder has similar prevalence between the sexes, but evidence suggests that men and women experience the disorder differently. Disorder onset, comorbidities, and treatment diverge significantly between men and women. For example, depressive episodes seem to predominate bipolar illness episodes in women, whereas in men, mania is more frequent [1]. There are also sex differences in management of medication side effects and comorbidities [2], and women with bipolar disorder report poorer sleep quality, which predicts more intense mood symptoms [3].

An oft-cited but poorly understood phenomenon in bipolar disorder is antidepressant-induced mania (AIM) or antidepressant emergent manic symptoms (AEMS). AIM describes the observation that hypomanic and manic symptoms can emerge when bipolar disorder patients use antidepressants, particularly when they are not taking a concurrent mood stabilizing medication. However, most bipolar disorder patients do not develop AIM when exposed to antidepressants, and the current data suggest that the risk factors for AIM in men and women may differ [4]. Clinicians routinely screen for AIM when treating bipolar depression with antidepressants, but little is known about which demographic and clinical variables increase risk for AIM, and even less is known about gender-specific risk factors.

In our retrospective study of 416 patients with bipolar disorders, we found that women were more likely to receive antidepressants than men [5]. This is in agreement with data from Karanti et al. [6] who showed that women with bipolar disorder were more likely to be prescribed antidepressants than men, which was independent of illness severity or other clinical factors. Strikingly, in our sample, female sex was the only variable that emerged from regression modeling as a statistically significant risk factor for AIM. In another recent study, Scott et al. [4] identified a number of factors that convey differential risk for AIM between men and women. They reported that male AIM patients were more likely to have an alcohol or substance use disorder, a history of suicide attempt(s), and a greater number of depressive episodes per year. Female AIM patients were more likely to have a history of thyroid disorder, family history of bipolar disorder type I, or a depressive episode at the onset of bipolar illness.

These early findings of differences between men and women with bipolar disorder, and how this may affect the incidence of AIM, are compelling and merit further study. Tools for identifying patients at highest risk would be very helpful, particularly since the incidence of AIM is low (generally estimated to be $\sim 10-20 \%$ of bipolar disorder patients who take antidepressants) but the dangers of mania are significant. It is unclear whether the observed treatment and outcome discrepancies between men and women with bipolar disorder are due primarily to physiological differences between sexes (e.g., hormonal pathways, sexspecific neuronal circuitry), or as described in Karanti et al [6], have less basis in physiology and more to do with disparities in how physicians treat women and men (Fig. 1).

\section{FUNDING:}

This work was supported by NIH UL1TR002240, the HC Prechter Bipolar Research Program and the Richard Tam Foundation of the University of Michigan, the Department of Psychiatry and Depression Center of the University of Michigan.

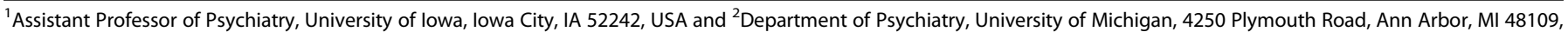
USA

Correspondence: Melvin G. McInnis (mmcinnis@umich.edu)

Published online: 28 September 2018 